\title{
Generic POLCA-A production and materials flow control mechanism for quick response manufacturing
}

\author{
Nuno Octávio Fernandes ${ }^{a}$; Sílvio do Carmo-Silva ${ }^{b}$ \\ a Instituto Politécnico de Castelo Branco, Departamento de Engenharia Industrial, Av. Do Empresário, 6000 Castelo Branco, \\ Portugal \\ ${ }^{\text {b }}$ Universidade do Minho, Departamento de Produção e Sistemas, Portugal
}

\begin{abstract}
A production and materials flow control mechanism for quick response manufacturing (QRM) is proposed. This is called generic paired-cell overlapping loops of cards with authorization (GPOLCA). It is an adaptation of the POLCA mechanism developed as part of the QRM strategy. GPOLCA implements an input-output control order release strategy based on an inventory of production authorization cards instead of materials. It is best suited for companies that manufacture large variety of products with variable demand. A description of GPOLCA is made together with a comparative study of its performance in relation with other mechanisms namely MRP and POLCA. The results show that GPOLCA attains better performance.
\end{abstract}

Keywords: Production control; Simulation; QRM; GPOLCA

\section{Introduction}

In today' s changing manufacturing world with new paradigms such as mass customization and global manufacturing operations and competition, companies need greater capabilities to respond quicker to market dynamics and varying demands.

The adoption of suitable production and materials flow control (PMFC) mechanisms, combined with the implementation of emergent technologies, can be of great value for improving performance and quality of manufacturing and of service to customers.

Quick response manufacturing (QRM) can be an effective competitive strategy for companies that work on a make-to-order (MT0) basis of large product variety ranges with variable demand, or even for companies that work on a engineering-to-order (ET0) basis (Suri, 1998). QRM focuses on reducing lead times throughout all the production supply chain activities of an organization. Externally, QRM means responding to costumers needs by providing quick design and manufacture of products. Internally, it means reducing lead times for all tasks, improving quality, reducing costs and increasing the speed of response. PMFC strategies for QRM must address the need and the capability of firms to respond quickly to highly changing product demand requirements. This is usually not possible with inventory replenishment strategies such as the one implemented by the Toyota Kanban System (TKS), (Suri, 1998). Paired cell overlapping loops of cards with authorization (POLCA) is a hybrid push-pull PMFC mechanism developed as part of the overall strategy of QRM.

According to Suri (1998), POLCA is a mechanism that overcomes the limitations of pull systems in high-variety and/or customization product environments, demonstrating its apparent relevance for the MTO and ETO sector. Suri (1998) uses the pull and push concepts as defined by Spearman and Zazanis (1992). These authors consider push systems as those that control throughput (TP) by establishing a master production schedule and measure work-in-process (WIP), and pull systems as those that control WIP and measure TP against the required demand.

In this paper, a new PMFC mechanism, called Generic POLCA (GPOLCA) is proposed, to address QRM under MTO and ETO environments. The performance of GPOLCA is evaluated and compared with MRP and POLCA in a multi-station multi-product manufacturing system, under changing product mix and highly variable demand conditions. 
The rest of the paper is organized as follows. In Sections 2 and 3 a classification scheme for PMFC mechanisms is presented, and the GPOLCA is described together with an analysis of some of its properties. In Section 4, GPOLCA is studied using computer simulation and its performance is compared with other PMFC mechanisms. Section 5 comprises the conclusions and directions for further work.

\section{Production and materials flow control mechanisms}

A PMFC mechanism refers to any procedure or process designed to control both, production and materials flow throughout a production system, i.e. from the acquisition of raw materials to the delivery of final products to customers. Good PMFC mechanisms must ensure good use of resources, i.e. production and materials handling resources, and short delivery times. PMFC mechanisms address two main functions: order release and materials flow control. Order release determines the time and the production orders (or jobs) to be released into the production system for production to start. Release decisions are usually based on order urgency and order influence on the current shop floor situation (Henrich et al., 2004). Once the job release function is triggered, the choice of which job or jobs to be released must be made based on order selection rules. A released job stays in production until all operations have been completed.

Materials flow control coordinates the flow of materials and production needs throughout the production process. This essentially involves work centre activation, i.e. the start of processing once materials are available and taking decisions for moving materials between work centres. In some mechanisms the availability of materials suffices for production to start. In other cases further control conditions must be satisfied for work centre activation. Clearly, dispatching rules are also important in the materials flow control process. In this context, once conditions for work centre activation are created, dispatching rules determine which orders or jobs in queue should be selected for processing. Nevertheless, experience has shown that dispatching is a relatively weak mechanism when used alone (Haskose et al., 2004), this clearly influences the progress of individual orders through the shop floor. A variety of well-known dispatching rules may be used for this end (Blackstone et al., 1982; Ramasesh, 1990).

Because of the great importance of PMFC for the competitiveness of manufacturing enterprises, many mechanisms have been developed. Graves et al. (1995), Liberopoulos and Dallery (2000) and Bergamaschi et al. (1997) review literature on several of these mechanisms.

\subsection{Classification based on order release strategy}

In relation to order release strategy PMFC mechanisms can be classified into five major classes, namely: immediate release, input, output, input- output and bottleneck control mechanisms. Immediate release (IMR), releases jobs to the shop floor immediately after demand occurs, without taking into account any information about the

system status or the characteristics of the jobs to be released. For instance, in the base stock mechanism (Kimball, 1988), which can be seen as a paradigmatic example of IMR, any order for finished items immediately triggers the release of work at each work centre, authorizing production to meet requirements for the finished items. The base stock mechanism works by keeping, at each work centre in the system, a certain amount of inventory called the base stock level.

In input PMFC mechanisms, materials are released into the system based on pre-defined release dates. These mechanisms schedule releases based on a master production schedule. Input control mechanisms consider only due dates, without taking into account the system status to release jobs into the system. Thus, when the time arrives, materials are released into the different stages of production and processing may start. Because of this, it can be said that these mechanisms control 
throughput and measure WIP. Material requirements planning- MRP (Orlicky, 1975), is an example of an input PFMC mechanisms. In MRP, the release times for different orders are established by backward scheduling due dates from estimated lead times for manufacturing and materials supply. Output PMFC mechanisms release work into the system based on the current consumption of inventory or on the completion of already released jobs. This is usually done by setting inventory or workload planned levels, for each work centre in the jobs' routings or for the production system as a whole. Output control mechanisms consider only the system status ignoring due dates. These mechanisms authorize releases through workload control, i.e. WIP control, measuring the resulting TP against the required demand.

The TKS (Sugimori et al., 1977) and the CONstant Work in Process (CONWIP) (Spearman et al., 1990) are examples of output PMFC mechanisms. In the TKS, when a unit of material is consumed from a work centre output buffer by a downstream work centre, the production of an identical unit can start for replacing consumption purposes. This can be repeated for every upstream supplying work centre, up to the first stage of production, establishing the timing and the job to be released. Kanbans set inventory planned levels for each part type at each work centre in the system. In the CONWIP mechanism, when a unit of material is consumed from the finished goods inventory, at the last stage of the production system, a new unit is released into the system. The released unit may, however, be different from the one consumed. This very much depends on the selection rule and the type of jobs waiting to be released into the system.

Under CONWIP, inventory planned levels are established for the whole production system on the basis of the number of CONWIP cards. Once released into the system, jobs are pushed through work centres together with CONWIP cards, to ensure, as much as possible, that the system bottleneck is kept busy. CONWIP cards are not part specific, i. e. can be allocated to any type ofpart.

It is important to note that some output control mechanisms are essentially based on inventory planned levels, as are the cases of TKS and CONWIP, and others are based on workload planned levels strategies, as is the proposed GPOLCA mechanism. The former may be classified as inventory replenishment mechanisms, while the latter as load-limited mechanisms. Load-limited mechanisms load the production system, or each work centre, with work up to the established workload levels or norms. Workload can be measured either in terms of the number of jobs or in terms of the amount of work (e. g. hours of work). Load-limited mechanisms ensure that each work centre only works on jobs for which capacity for further processing, in downstream production stages, has been reserved. These mechanisms are most adequate for MT0 or ET0 manufacturing environments.

Inventory replenishment mechanisms are based on inventory-planned levels, where the release of new jobs into the system is triggered by the current consumption of inventory, i.e. jobs that have been removed from inventory to satisfy demand. These mechanisms are likely to be more useful in a repetitive production environment where workload is usually measured in terms of the number of jobs.

Input- output PMFC mechanisms combine features of the input and output control. The release of orders into the system is dependent on both, release dates and production authorizations, usually in the form of cards. Once these two conditions are met and as long as the manufacturing resources required are available, production can start.

Synchro-MRP (Hall, 1981) and POLCA (Suri, 1998) are examples of input-output PMFC mechanisms.

Both use MRP for establishing allowed release dates, for job processing in each work centre or cell.

POLCA sets workload planned levels for each pair of cells while Synchro-MRP sets inventory planned levels in a way similar to the TKS. Release dates are not the only 
factor that needs to be taken into account for allowing the release of orders, and subsequent production. Kanbans and POLCA cards are also necessary for Synchro-MRP and POLCA, respectively. The proposed GPOLCA mechanism can also be classified as an input-output control mechanism.

In bottleneck PMFC mechanisms when a job is completed by a bottleneck work centre, a new jobwith similar workload at the bottleneck, is released into the system. The released unit may, however, be different from the one completed. This depends on the selection rule and on the type of jobs waiting to be manufactured. Thus, the rate of jobs release is determined by the production rate at the bottleneck.

It is important to ensure that at the input buffer of the bottleneck work centre there is always an inventory of work to be processed. The purpose of this inventory is to keep the bottleneck busy, towards maximum throughput. The drum-bufferrope (DBR) mechanism proposed by Goldratt and Fox (1986) and the starvation avoidance (SA) policy (Glassey and Resende, 1988) developed for the semiconductor industry are paradigmatic examples of bottleneck control. Bottleneck PMFC mechanisms may share some of the characteristics of input or output control mechanisms or both. But it is its distinctive nature of linking bottlenecks to the order release process that distinguishes them. Thus, for example, on the one hand the DBR mechanism uses, for order release, knowledge about the bottleneck status, namely its inventory consumption, and usually requires a detailed schedule, based on bottlenecks, to be built in advance; on the other hand, the SA policy referred above, does not require release dates to be defined.

\subsection{Classification based on the materials flow control strategy}

As far as materials flow control is concerned, most of the known PMFC mechanisms can be classified as push and pull mechanisms.

Frequently, the existence of materials is sufficient for work centre activation. On the completion of jobs in one work centre, they are simply pushed into the next one for further processing, until all operations have been completed. In this case, the system is operated with a push materials flow control mechanism, i. e. the system is operated to carry out production on a machine whenever there is material to be worked on, with no regard to the amount of inventory downstream. On the other hand, in a pull materials flow control mechanism, the production in a particular work centre is triggered by the demand of materials from downstream work centres. Pull systems are operated to keep a machine idle, even when work is waiting, until a signal from downstream authorizes this machine to produce. It must be emphasized that the pull and push concepts adopted in this work are coincident with those expressed by Tayur (1993) and are directly related with the materials flow control strategy, whilst those presented by Spearman and Zazanis (1992), also frequently accepted, are different, and essentially related to order release strategy.

Table 1 is a summary of the classification of the PMFC mechanisms.

\section{The GPOLCA mechanism}

GPOLCA is a PMFC mechanism for QRM that adapts ideas from the POLCA mechanism. Like POLCA, it controls order release through a combination of release dates and production authorization cards allocated to pairs of cells.

However, although implementing an input- output control order release strategy like POLCA, GPOLCA does this based on an inventory of production authorization cards instead of on the POLCA inventory of materials strategy. Moreover, whilst

P0LCA implements the pull materials flow control strategy, GPOLCA implements the push type control strategy subject to the reserved capacity associated to production authorization cards. 


\subsection{Mode of operation}

GPOLCA uses cards to control the number of jobs, i.e. WIP, in the system. These cards are not specific to any particular part type and are allocated to specific pairs of work centres or cells, preferably product oriented cells, like group technology

cells, see Fig. 1. The same card can be reused and allocated to different jobs, as long as they need processing in the same particular pair of cells. GPOLCA cards set workload levels for each pair of cells and consequently for the whole system. By setting the number of GPOLCA cards the WIP for each pair of manufacturing cells

is controlled. An inherent characteristic of GPOLCA is that it ensures that a given cell only works on jobs if there is reserved capacity on all downstream cells.

Fig. 1 illustrates the GPOLCA card flow for a particular job or order in a manufacturing system.

GPOLCA cards must be available in order to be allocated to jobs. Starting from the last cell in the routing of a job order, successive allocations of cards to the order, in the upstream pairs of cells, ensure that capacity is reserved to allow the release of jobs into system. Once released, no order is restrained from being processed and pushed through the supply-production chain.

The flow of jobs and cards under GPOLCA control must comply with the following controlling

rules:

Rule 1. The required GPOLCA card, or cards, from each pair of cells or work centres, in a job routing, are simultaneously allocated to the job when it is released in the system. More than one card may be needed to allocate to a job, in each pair of cell, depending of the workload associated with the GPOLCA card.

Rule 2. On the arrival of a job to a cell, job processing can start immediately, as long as all required resources and materials are available.

A dispatching rule or other scheduling mechanism should be used to decide which job to process first.

Rule 3. GPOLCA cards stay with a job from the moment it is released, until the moment the customer (or downstream) cell, in the pair of cells, completes the processing of the job.

Rule 4. After job completion at the customer cell, the allocated card becomes available for allocation to another job. Rules above show an underlying characteristic of GPOLCA, non-existent in POLCA. That is, GPOLCA releases jobs only after the GPOLCA cards, necessary by a job at each pair of cells, in the job' $\mathrm{s}$ routing, become available and are allocated to the job. POLCA, on the other hand, undertakes the job release as long as cards for the first pair of cells, in

the job' s routing, are available.

In GPOLCA a card belonging to a pair of cells, for example, C1-C2 (see Fig. 1) is, therefore, attached to a job when it is released into the system.

Then, it stays with the job through the pair of cells, C1 and C2 in this case, and with job completion on the second cell of the pair, i.e. C2, the card becomes available to be allocated to another job. In POLCA there are differences. In fact, a card belonging to a pair of cells, for example C1-C2, will be attached to a job when it enters the first cell of the pair, $\mathrm{C} 1$ in this case. It then stays with the job, even after it has been completed in the second cell of the pair, i.e. C2, until it enters the next cell in the routing, i.e. cell C3 in the case of Fig. 1. Only then the card becomes available for allocation to another job.

Orders arrive over time to the production system and may have to wait in a backlog or pool of orders

to be released according to GPOLCA control (see Fig. 1). The GPOLCA procedure to release an order into a manufacturing system is as follows:

Step 1: For each order in the pool, a planned release date is determined by backward scheduling from the due date, using the planned lead times. 
Step 2: Each order is considered for release according to the earliest planned release date.

Step 3: Order release takes place when the required GPOLCA cards from each pair of cells, in the routing of the job order, are all simultaneously available to be allocated to the order.

Step 4: Each unreleased order waits in the pool until the workload in the required pairs of cells falls below a planned level, i.e. until enough GPOLCA cards become available.

\subsection{Parameters}

The parameters established in the GPOLCA mechanism are:

$\mathrm{c}_{1}, \quad \mathrm{c}_{2}, \quad y, \quad \mathrm{c}_{\mathrm{n}}$ Represents the number of GPOLCA cards in each pair of cells. This sets the maximum WIP and influences job flow times and system TP.

$\mathrm{q}$ Is the amount of workload that a GPOLCA card represents. This is necessary in order (a) to establish the right number of GPOLCA cards to be assign to each pair of cells based on workload planned levels, and (b) to calculate the number of cards that will be necessary to allocate to each job or order. In practice, due to different capacity requirements of jobs, a different number of cards is likely to be allocated to each job in each pair of cells.

Ts Represents the planned lead time for each cell used within the release procedure to determine de planned release dates $\mathrm{t}_{\mathrm{j} r}$ for each job $\mathrm{j}$. Thus, assuming $\delta_{\mathrm{j}}$ as the due date of the job $\mathrm{j}$ and $\mathrm{S}_{\mathrm{j}}$ as the set of cells in the jobs routing, then

$$
t_{j}^{r}=\delta_{j}-\sum_{s \in S_{j}} T_{s} .
$$

Little (1961) describes, from a theoretical point of view, the relationship between WIP, manufacturing lead time (MLT) and TP, and points out that there is a critical level of WIP, i.e. workload, which should not be exceeded in a manufacturing system. This is because, when WIP increases beyond this level, it leads to an increase in flow times and consequently in MLT, without any meaningful improvement in TP.

Good GPOLCA parameter choices are those that attain high TP at low WIP. TP tend to increase as the number of cards increase. However, the number of cards can be increased up to the level where TP no longer increases. As long as the number of cards is big enough, TP is only restricted either by demand or by the system capacity.

\subsection{GPOLCA properties}

\subsubsection{Manufacturing environment}

GPOLCA is particularly suited for MTO and ET0 environments and even though manufacturing system configuration should preferably be of the flow-shop type, other configurations, including job shop, can be considered. The reason is because the cards setting for the GPOLCA mechanism is easier to establish in flow-shop type configurations than in the job-shop ones. The number of pairs of cells, which are defined by the routings of jobs, tends to be larger in job-shops than in flow shops. Because of this, companies should preferably have a product oriented approach to manufacturing, with work centres or cells arranged according a predominant direction of the flow of materials.

\subsubsection{Input- output control release strategy}

GPOLCA is an input- output mechanism, i.e. has both input and output control characteristics. Under GPOLCA control, orders are released into the production system based on planned release dates and production authorizations, i.e. GPOLCA cards. These set workload planned levels, for each pair of cells in the jobs routing. 


\subsubsection{Load limitation}

GPOLCA can be seen as a zero-inventory system in the sense that no replenishment of inventory, both, parts and end items, is allowed. The basic idea is to maintain an inventory of production authorization cards, for limiting the load in the system, instead of an inventory of materials. This makes the GPOLCA particularly suited to the manufacturing environment referred above and distinguished itself from the inventory replenishment mechanisms which are suitable for repetitive production under stable demand and a levelled master schedules.

\subsubsection{Push materials flow control strategy}

Under GPOLCA, once materials are released into the system they are pushed from one cell to another, through the several stages of processing, restricted only by production capacity. This means that GPOLCA has a push characteristic.

\section{Simulation study}

In order to compare alternative PMFC mechanisms, computer simulation was used to evaluate performance under a given production system setting. The performance results of GPOLCA were compared with those of two other PMFC mechanisms, namely MRP and POLCA.

\subsection{Production system and simulation setup}

The simulation study was carried out using a production setting similar to that used by Krishnamurthy et al. (2004), where these authors undertook a comparative study between the TKS and MRP performance. The physical and the operational production system configuration include:

1. A multi-product manufacturing line with three work centres $\mathrm{j}=1,2$ and 3 .

2. A single machine in each work centre.

3. Demand arrival rate is distributed according to a Poisson process.

4. Service times for product model $\mathrm{i}$ at work centre $\mathrm{j}$ are exponentially distributed.

5. Set-up times are negligible on all machines and for all jobs.

6. First-come-first-served (FCFS) job dispatching rule is used in all work centres.

7. Material is transported in units of one, and it is assumed that there are no delays.

8. Information related to the handling GPOLCA cards is transmitted instantly. Exponential and Poisson distributions were assumed for service times and demand arrival, respectively, in order to model a high variable environment.

The performance measures used are TP, WIP and Flow time. More specifically, the objective is to compare the minimum average WIP required to obtain a desired value of TP. WIP is defined as the amount of material that has been actually loaded into the first work centre, but not yet delivered to the customer. This is calculated as the sum of the mean inventory levels at each work centre of the production system. TP is measured as the mean number of the completed products per time unit.

Flow time, which is sometimes also referred to as cycle time, is considered to be the time between the job release and its completion. Service times, $\mu_{\mathrm{ij}}$, for the two product models considered, $\mathrm{i}=1$ and 2 , at all work centres, are made equal to one, and the line is well balanced. Based on this, simulation experiments were conducted for release rates, $\lambda_{i}$, varying from 0.5 to 0.95 . For the MRP mechanism, the release rate is the only control parameter considered. In this case, for each value of TP the corresponding WIP was measured.

For POLCA and GPOLCA mechanisms, the control parameters are the release rate and the number of cards. A search is carried out, to find the minimum number of cards in each pair of work centres which leads to the desired TP. At this point the value of the WIP in the system is measured. 
Parts that are authorized for loading at the first work centre are not considered until they are actually loaded. Parts waiting to be loaded are not accounted in any of the mechanisms considered.

The performance measures referred before represent the mean values of 50 independent identically distributed replications with run length of 96, 000 time units. An initial warm up period of 9600 time units was used to nullify transient start-up effects.

\subsection{Performance results and discussion}

For each performance variable analysed, resulting from experiments, namely WIP, TP and Flow Time, the Kolmorov-Smirnov test to normality was carried out. No statistical evidence was detected for rejecting the null hypothesis at 95\% confidence level ( $\mathrm{p}$ value $<0.05$ ). Based on this, the 95\% confidence intervals were developed, for the mean value of each performance measure, using the t-student distribution (see for example Law and Kelton 2000, p. 253).

\subsubsection{Performance under homogeneous product mix}

In the first set of experiments, the performance of the system was compared when subject to variable demand and homogeneous product mix, whilst operating under MRP, POLCA and GPOLCA

PMFC mechanisms can be compared based on the mean TP vs. mean WIP trade-off curve. This curve describes the average value of WIP as a function of the system throughput, (Fig. 2). It is known that as throughput approaches the capacity of the system, i.e. the maximum possible system throughput, the average WIP tends to infinite. In Fig. 2, WIP is plotted against TP for the three mechanisms tested. A point on the curve corresponds to the parameters choice, for a particular mechanism, that achieved that TP with the least WIP.

It can be said that a particular PMFC mechanism is superior to another if, for a given TP it shows a lower WIP. To investigate this further, a configuration that gives a throughput of 0.95 using the least possible WIP was considered.

Table 2 shows the performance results of the three mechanisms, for the above TP objective.

Significant differences in the performance results of the three mechanisms are identified on the basis of paired t-tests with 95\% confidence level.

Analysing the above results the following observations can be made:

- In this environment, the input-output control mechanisms POLCA and GPOLCA perform better than input control mechanism MRP. The total WIP required to meet a particular TP is higher in the MRP mechanism, particularly for high levels of TP.

- Results under GPOLCA are clearly better than under MRP and improve as the system TP increases. For a TP of 0.95, changing from MRP to GPOLCA produced a reduction in WIP of 26.5\%. This means that the same TP can be obtained under GPOLCA with less WIP and lower flow time, as shown in Table 2, as could be expected by the Little' s law.

- Over a large range of TP values, GPOLCA and POLCA have identical performance rates. However, at the highest levels of TP, GPOLCA performs better than POLCA. In fact, for the stated TP objective, changing from POLCA to GPOLCA reduces WIP in $6.6 \%$.

For the single product case the superiority of TKS in relation to MRP has been reported by Spearman et al. (1990). For multi-product environments under homogenous product mix, Krishnamurthy et al. (2004) found that input control mechanisms like MRP, (according to our classification), perform better than output control mechanisms TKS, in terms of service level and average WIP. These authors suggest that this performance behaviour is due to the "resident" WIP, i.e. the minimum WIP inventory that is kept by the TKS for each product in the system. Although MRP may perform 
better than TKS for the multi-product case, the results in this paper show that GPOLCA and POLCA perform better than MRP.

\subsubsection{Performance under changes in product mix}

In this section a set of experiments was conducted in order to compare system' $\mathrm{s}$ performance in the presence of short-term imbalances caused by product mix changes. In particular, the change in the system' $s$ TP, as a result of changes in the relative ratios of mean release rate and mean service times, was studied. These changes take place over a short period of time, i. e. during 1 day per week. Over this period the allocation of cards in each pair of work centres is kept unchanged.

For comparison purposes, the total demand for the two models, $\lambda_{1}+\lambda_{2}=1$ was considered and a demand rate ratio $1_{1} / l_{2}$ of $1 / 2$ and a service time ratio $\mu_{1} / \mu_{2}$ of 2 were assumed. The expected mean service time in each work centre is set to one (Eq. (2)). This setting ensures that the manufacturing line stays balanced:

$$
\frac{\lambda_{1} \mu_{1}+\lambda_{2} \mu_{2}}{\lambda_{1}+\lambda_{2}}=1
$$

In Fig. 3, WIP is plotted against TP for each of the mechanisms tested. As it can be seen, in the presence of product mix changes, the performance of all the mechanisms deteriorates, i. e. WIP increases for the same levels of TP.

Table 3 shows the performance of each mechanism that achieves the sated TP objective. A paired ttest reveals that, the difference between each WIP and flow time level of GPOLCA and each WIP and flow time level from each one of the other mechanisms is significant at $95 \%$ confidence level.

Analysing the above results the following new observations can be made:

- Input-output control mechanisms again show better TP than the input MRP mechanism, for identical values of WIP. Consistently MRP performs worse than POLCA and GPOLCA mechanisms.

- The behaviour of GPOLCA in relation to the other mechanisms has clearly improved under this product mix changes setting. In fact for a TP of 0.95 , changing from POLCA to GPOLCA produces a WIP reduction of $11.3 \%$, while changing from MRP to GPOLCA reduces WIP by 27.6\%. As expected, a similar reduction in job flow time is also noticeable.

- Whilst the performance of all mechanisms deteriorates as a consequence of the increased variability, it deteriorates less under GPOLCA control. Actually, under GPOLCA WIP deteriorates less than $1.5 \%$ for this new experimental setting, while under POLCA and MRP it deteriorates by $6.8 \%$ and $3.0 \%$, respectively.

This indicates that, as product mix changes, GPOLCA' s performance is likely to deteriorate less than the other two mechanisms, and the POLCA is the mechanism for which performance deteriorates most.

From the above experiments we can conclude that, GPOLCA performs better than POLCA. Apparently, the qualities of GPOLCA are emphasized under dynamic production environments, with changes in the product mix. The strategy of holding an inventory of production authorization cards, instead of an inventory of materials, has a significant effect on reducing WIP.

Fig. 4 shows the distribution of WIP across the work centres of the system. It is clear that GPOLCA and MRP have a similar pattern. This seems to be due to the fact that both implement a push materials flow control strategy. However, much lower values of WIP are obtained under GPOLCA.

Considering that POLCA control is seen as a highly performing mechanism for QRM, the results obtained in this paper for the GPOLCA mechanism can be considered important. 


\section{Conclusions}

PMFC mechanisms are becoming more and more important, not only under repetitive production but also under today' s highly varying market demand environments and globalization. The reason is because they can contribute for better performance, through better control of production and materials flow, over the whole production system, let it be localized or distributed in a regional or global space.

However, it is clear from many studies on the subject that under different market demand environments, different PMFC mechanisms must be used for efficiently running operations.

In this paper, in addition to the proposed classification scheme for PMFC mechanisms we also propose a new PMFC mechanism for QRM, called GPOLCA.

The performance of GPOLCA was compared with the performance of POLCA and MRP mechanisms in a multi-stage, multi-product manufacturing system, under changing product mix and highly variable demand. We find that for this environment, GPOLCA outperform both mechanisms, ensuring the same high level of throughput with less WIP. Furthermore, GPOLCA showed to be more robust to changes in the product mix.

Many benefits of GPOLCA can be attributed to the fact that it is a mechanism with a push materials flow control nature, and to the fact that maintaining an inventory of production authorization cards, instead of an inventory of materials, seems to have a significant effect on reducing the WIP mainly for high levels of TP.

GPOLCA is not necessarily the best form of controlling production and materials flow in every manufacturing environment. However, it seems to be specially suited for today' s marketplace, where we can find an increasing demand for customized products. Future research should determine if the results for the environment considered in this paper, hold for other production control conditions and shop floor operation conditions.

\section{References}

- Bergamaschi, D., Cigolini, R., Perona, M., Portioli, A., 1997. Order review and release strategies in a job shop environment: A review and classification. International Journal of Production Research 35 (2), 339-420.

- Blackstone, J.H., Phillips, D. T., Hogg, G. L., 1982. A state of the art survey of dispatching rules for manufacturing job shop operations. International Journal of Production Research 20 (1), 27-45.

- Glassey, C.R., Resende, M. G. C., 1988. Closed 1oop job release control for VLSI circuit manufacturing. IEEE Transactions on Semiconductor Manufacturing 1 (1), 36-46.

- Goldratt, E. M., Fox, R.E., 1986. The Race. North River Press, New York.

- Graves, R. J., Konopka, J.M., Milne, R. J., 1995. Literature review of materials flow contro1 mechanisms. Production Planning and Control 6 (5), 395-403.

- Hall, W. R., 1981. Driving the productivity machine, Production Planning and Control in Japan. (Falls Church, Virginia) American Production and Inventory Control Society.

- Haskose, A., Kingsman, B. G., Worthington, D., 2004. Performance analysis of make-to-order manufacturing systems under different workload control regimes. International Journal of Production Economics 90 (2), 169-186.

- Henrich, P., Land, M., Gaalman, G., 2004. Exploring applicability of the workload control concept. International Journal of Production Economics 90 (2), 187-198.

- Kimba11, G., 1988. General principles of inventory control. Journal of Manufacturing and Operations Management 1 (1), 119-130.

- Krishnamurthy, A., Suri, R., Vernon, M., 2004. Re-examining the performance of MRP and Kanban material control strategies for multi-product flexible manufacturing systems. International Journal of Flexible Manufacturing Systems 16, 123-150.

- Law, A. M., Kelton, W. D., 2000. Simulation Modelling and Analysis, 3rd ed. McGraw Hi11, Singapore.

- Liberopoulos, G., Dallery, Y., 2000. A unified framework for pull control mechanisms in multistage manufacturing systems. Annals of Operation Research 93, 325-355.

- Little, J. D. C., 1961. A proof for the queuing formula: L1/4 1W. Operations Research 9, 383-387.

- Orlicky, J., 1975. Materials Requirement Planning. McGraw Hi11, New York.

- Ramasesh, R., 1990. Dynamic Job shop scheduling: A survey of simulation research. 0mega 18 (1), 43-57.

- Spearman, M. L., Zazanis, M. A., 1992. Push and pul1 production systems: Issues and comparisons. Operations Research 40 (3), 521-532. 
- Spearman, M. L., Woodruff, D. L., Hopp, W. J., 1990. Conwip: A pull alternative to Kanban. International Journal of Production Research 28 (5), 879-894.

- Sugimori, Y., Kusunoki, K., Cho, F., Uchikawa, S., 1977. Toyota production system and Kanban system materialization of just-in-time and respect-for-Human system. International Journal of Production Research 15 (6), 553-564.

- $\quad$ Suri, R., 1998. Quick Response Manufacturing: A Company-Wide Approach to Lead Time Reduction. Productivity Press, Portland.

- Tayur, S. R., 1993. Structural properties and a heuristic for kanban-controlled serial lines. Management Science 39 (11), 1347-1368. 
Tables

Table 1

Classification of PMFC mechanisms

\begin{tabular}{lll}
\hline $\begin{array}{l}\text { Order release } \\
\text { strategy }\end{array}$ & \multicolumn{2}{l}{ Materials flow control strategy } \\
\cline { 2 - 3 } & Push control & Pull control \\
\hline IMR & & Base stock \\
Input & MRP & \\
Output & CONWIP & TKS \\
Input-output & GPOLCA & POLCA; Synchro- \\
Bottleneck & DBR; SA & MRP \\
\hline
\end{tabular}

Table 2

Performance under homogeneous product mix for a throughput of 0.95

\begin{tabular}{llll}
\hline Mechanism & TP & WIP & Flow time \\
\hline MRP & $0.95 \pm 0.0037$ & $38.58 \pm 0.96$ & $40.60 \pm 0.98$ \\
POLCA & $0.95 \pm 0.0032$ & $30.37 \pm 0.39$ & $31.97 \pm 0.40$ \\
GPOLCA & $0.95 \pm 0.0036$ & $28.37 \pm 0.34$ & $29.87 \pm 0.33$ \\
\hline
\end{tabular}

Table 3

Performance under product mix changes for a throughput of 0.95

\begin{tabular}{llll}
\hline Mechanism & TP & WIP & Flow time \\
\hline MRP & $0.95 \pm 0.0032$ & $39.74 \pm 1.22$ & $41.81 \pm 1.26$ \\
POLCA & $0.95 \pm 0.0026$ & $32.43 \pm 0.38$ & $34.14 \pm 0.39$ \\
GPOLCA & $0.95 \pm 0.0030$ & $28.77 \pm 0.35$ & $30.31 \pm 0.35$ \\
\hline
\end{tabular}




\section{Figures}

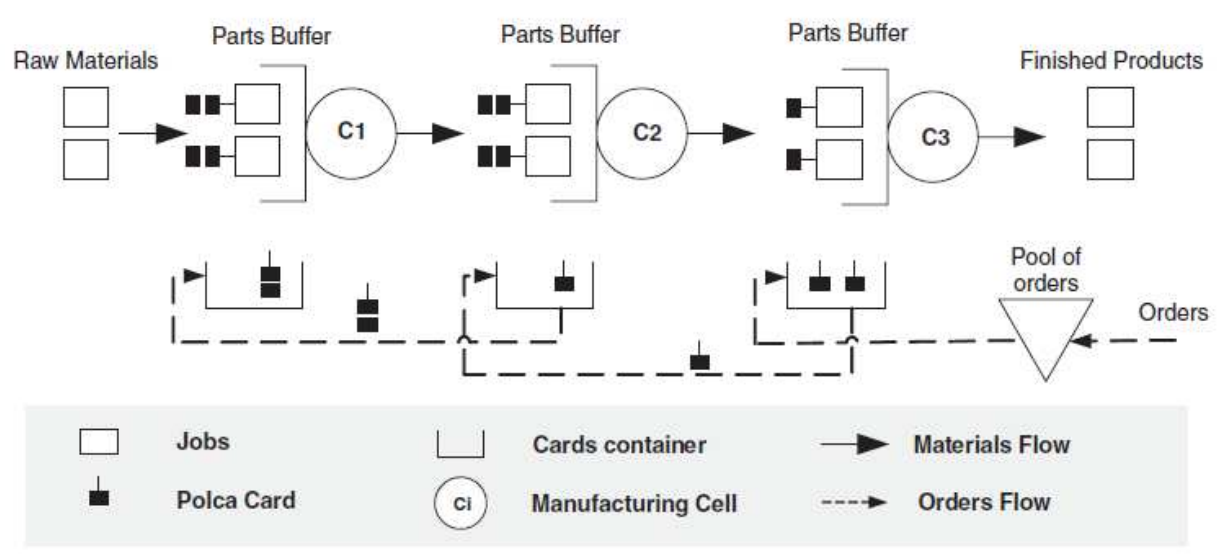

Fig. 1. Illustration of the GPOLCA mechanism.

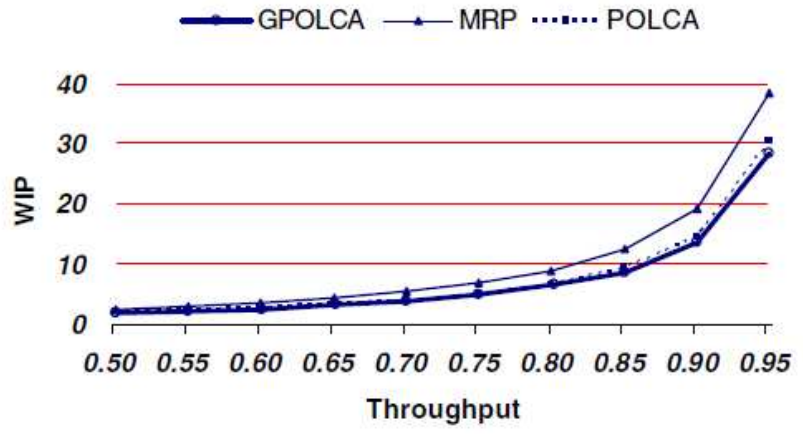

Fig. 2. Throughput vs. WIP under homogeneous product mix. 


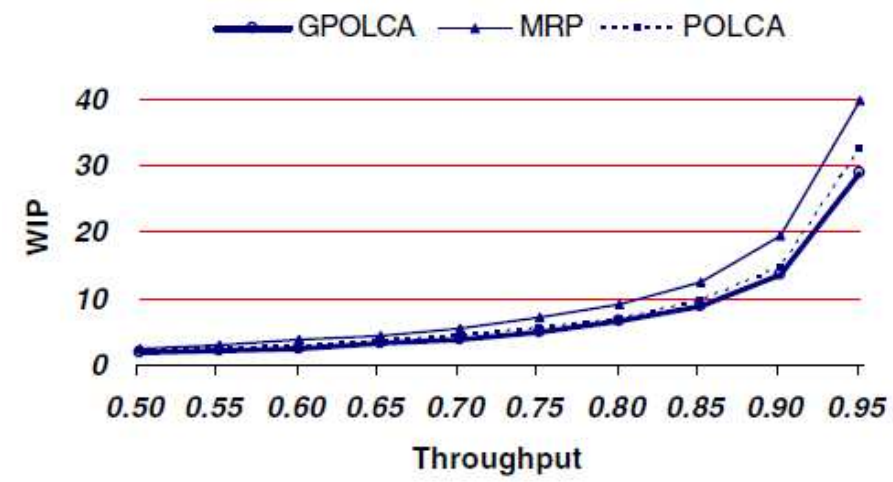

Fig. 3. Throughput vs. WIP under changes in the product mix.

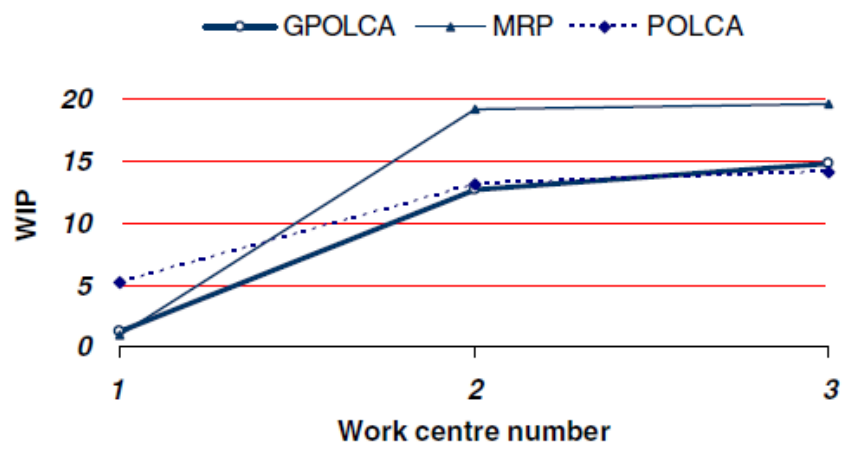

Fig. 4. Location of inventory. 